\title{
Clinical efficacy of intravenous anesthesia on breast segmental surgery and its effects on oxidative stress response and hemodynamics of patients
}

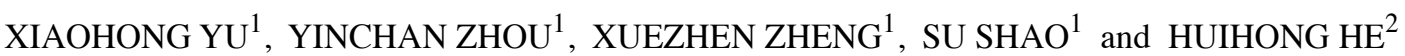 \\ ${ }^{1}$ Department of Nursing Care, Chun'an First People's Hospital; ${ }^{2}$ Department of Nursing Care, \\ Chun'an Hospital of TCM, Hangzhou, Zhejiang 311700, P.R. China
}

Received October 21, 2019; Accepted August 3, 2020

DOI: $10.3892 /$ etm.2020.9443

\begin{abstract}
This study was designed to investigate the clinical efficacy of intravenous anesthesia on breast segmental surgery and the effects on hemodynamics of patients. A total of 267 patients were collected as research subjects. These patients underwent breast segmental surgery in Chun'an First People's Hospital from March 2015 to September 2018. Among them, 137 patients undergoing intravenous anesthesia were the research group, and 130 patients undergoing inhalation anesthesia were the control group. The following parameters were recorded: Clinical efficacy, postoperative adverse conditions, hemodynamic indicators including systolic blood pressure (SBP), diastolic blood pressure (DBP) and heart rate (HR). Visual analogue scale (VAS) was used to observe the analgesic effect of the two groups, the mental state of patients in the two groups was observed by mini-mental state examination (MMSE) scoring method, and systemic evaluation was made by oxidative stress $(\mathrm{OS})$ reaction indicators. The MMSE scores of the two groups decreased one day after surgery, but the score in the research group was higher than that in the control group $(\mathrm{P}<0.05)$. The levels of SBP and DBP at $\mathrm{T} 1$ and $\mathrm{T} 2$ in the control group were significantly higher than those in the research group $(\mathrm{P}<0.05)$. HR of research group at $\mathrm{T} 1$ and $\mathrm{T} 2$ was lower than that at $\mathrm{T} 0$ and that at corresponding time of control group $(\mathrm{P}<0.05)$. The incidence rate of postoperative adverse reactions in the research group was significantly lower than that in the control group $(\mathrm{P}<0.05)$. In conclusion, intravenous anesthesia for breast segmental surgery can reduce the occurrence of adverse reactions after surgery, with complete sedation and analgesia. Patients were able to wake up quickly
\end{abstract}

Correspondence to: Dr Huihong He, Department of Nursing Care, Chun'an Hospital of TCM, 1 Xin'an West Road, Hangzhou, Zhejiang 311700, P.R. China

E-mail: hvk11k@163.com

Key words: intravenous anesthesia, inhalation anesthesia, breast segmental surgery, clinical efficacy, hemodynamics, oxidative stress response, cognitive function and stably after surgery, and their cognitive function and OS recovered rapidly. However, due to the great impact on hemodynamics during surgery, attention should be paid to maintain hemodynamic stability during surgery to avoid hypotension and bradycardia.

\section{Introduction}

Segmental breast surgery is one of the common methods in treating breast diseases. Clinically, general anesthesia is the main anesthesia method for breast segmental surgery, which can relieve pain, fear, anxiety and other negative emotions of patients. It is an important part of clinical study to ensure the anesthetic effect while shortening the postoperative recovery time and reducing anesthesia-related complications. Intravenous anesthesia has advantages such as rapid onset, strong efficacy, reversible anesthetic effect, and full variety of drugs. Compared with inhalation anesthesia, it does not burn, explode or pollute the operating room environment. Intravenous anesthesia has gradually become the mainstream technique of clinical anesthesia (1).

The effect of general anesthesia drugs on oxidative stress (OS) and hemodynamics of patients during anesthesia is currently a hot issue. Therefore, it is of great significance to compare the perioperative OS response of patients from the perspective of general anesthesia drugs for the selection of clinical anesthesia drugs. Intravenous anesthesia effectively maintains the stability of patients' hemodynamics, reduce the anesthetic dosage, inhibits the occurrence of OS with higher efficacy, and helps patients wake up quickly and safely from anesthesia (2). OS reaction refers to the oxidative damage process in vivo or in cells, which is caused by the imbalance between the generation and elimination of oxygen radicals in vivo or in cells, resulting in accumulation of reactive oxygen species (ROS) and reactive nitrogen species (RNS) (3). Recent studies have shown that DNA damage and genetic mutation are all related to OS. It can also promote and lead to the occurrence of tumors (4-7). Moreover, OS reaction is also regarded as an important factor leading to aging and diseases, because of a series of negative effects produced by free radicals in vivo or in cells (8). Because of the strong oxidizing ability, ROS could easily form negative ions by combining with one 
electron. According to different sources, ROS can be roughly divided into exogenous and endogenous ROS (9). Excessive ROS in the body would accelerate the aging of the body, while endogenous antioxidant enzymes [superoxide dismutase (SOD), catalase (CAT), glutathione peroxidase (GSH-Px)] are the natural barriers between cells and plasma, which can promote the reactions of various proteins, microorganisms and enzymes in the body, and inhibit or transform the generation of $\operatorname{ROS}(10,11)$.

This study investigated the clinical efficacy of intravenous anesthesia on breast segmental surgery and its effects on hemodynamics by detecting hemodynamic parameters [systolic blood pressure (SBP), diastolic blood pressure (DBP), heart rate (HR)], OS response indicator and other data of patients in the two groups with breast segmental surgery under intravenous anesthesia and inhalation anesthesia.

\section{Patients and methods}

General information. A total of 267 patients were selected, who underwent breast segmental surgery in Chun'an First People's Hospital (Hangzhou, China) from March 2015 to September 2018. Among them, 137 patients under intravenous anesthesia were the research group, with an average age of $40.75 \pm 6.88$ years. There were 62 patients with hypertension, 55 patients with hyperlipidemia, 31 cases with breast cancer, 36 cases of breast fibroids, 37 cases with benign breast masses and 33 cases of localized cystic hyperplasia of the breast. The 130 patients who received inhalation anesthesia were the control group, with an average age of $39.87 \pm 6.31$ years. There were 58 patients with hypertension, 60 patients with hyperlipidemia, 29 cases of breast cancer, 34 cases of breast fibroids, 36 cases of benign breast masses and 31 cases of localized cystic hyperplasia of the breast.

Inclusion criteria: Patients accompanied by family members upon admission, patients aged between 30 and 50 years, those with education level of primary school or above, complete clinical data and good compliance, and voluntary cooperation on follow-up investigation; patients in Grades I-II of the American Society of Anesthesiologists (ASA), with the operation time between 30 to $50 \mathrm{~min}$.

Exclusion criteria: Patients with unconsciousness; patients allergic to narcotic drugs; patients with severe organic diseases, patients who were unable to cooperate with the examination due to other factors such as aphasia, dysphoria and other communication disorders.

This study was approved by the Ethics Committee of the Chun'an First People's Hospital. All patients and their families were informed prior to the study and provided a signed complete informed consent.

Anesthesia methods. Patients fasted for $8 \mathrm{~h}$ before surgery and were forbidden to drink $6 \mathrm{~h}$ before surgery. Before anesthesia, the venous access on one side of the patient was normally opened, and routine items such as electrocardiogram (ECG), respiratory rate (RR), (SpO2), SBP, DBP, and HR were monitored without any pre-anesthesia drugs. Propofol (Shanghai Yuanye Biotechnology Co., Ltd.; B33792-100 mg) 1-2 $\mathrm{mg} / \mathrm{kg}$ combined with remifentanil (Shanghai KE WEI CHEM; R143501) $0.5 \mu \mathrm{g} / \mathrm{kg}$ was used to induce anesthesia in the research group, and propofol $4 \mathrm{mg} / \mathrm{kg} / \mathrm{h}$ combined with remifentanil $12 \mu \mathrm{g} / \mathrm{kg} / \mathrm{h}$ was used for anesthesia maintenance. The control group was anesthetized with $6 \%$ sevoflurane (YKPPSJ-009985) volatilization tank and $61 / \mathrm{min}$ oxygen flow rate, and anesthesia was maintained with $2-4 \%$ sevoflurane volatilization tank and 1-2 1/min oxygen flow rate. Then the position of laryngeal mask was determined and inserted, the respiratory frequency was adjusted to $12-16$ times $/ \mathrm{min}$ and the tidal volume to $6-10 \mathrm{ml} / \mathrm{kg}$. After surgery, the extubation time was recorded, and then the patients were sent to PACU for awakening. The awakening time of patients, the number of dysphoria in the awakening period and eye-opening time were recorded. If the patient had hypotension during surgery, $6 \mathrm{mg}$ ephedrine was given intravenously, and $0.5 \mathrm{mg}$ atropine was given intravenously for bradycardia.

Assay methods. Enzyme activity determination: 5-8 ml central venous blood was extracted from patients at different time points, T before surgery, T6, T24, T48 and T72 h after surgery. The collected venous blood was placed in heparin sodium blood collection vessel, centrifuged at 1,369.55 x g, at $4^{\circ} \mathrm{C}$ for $8 \mathrm{~min}$ to separate serum, and the separated serum was stored at $-80^{\circ} \mathrm{C}$ for later testing. SOD, CAT and GSH-Px activities were determined. The determination method was in accordance with the description in the kit (WST-1 method was used to determine SOD activity, visible spectrophotometry was used to determine CAT activity, and colorimetry was used to determine GSH-Px activity). SOD kit: Shanghai Yubo Biotechnology Co., Ltd., IC-SOD-Ra; CAT kit: Shanghai Jingkang Bioengineering Co., Ltd., JKSJ-1907; GSH-Px kit: Shanghai Jingkang Bioengineering Co., Ltd., JK-EA00285.

Main instruments and equipment. Main instruments and equipment were as follows: Multi-functional ECG monitor (Shanghai Hanfei Medical Equipment Co., Ltd.; BSM-3763), anesthesia machine (Beijing First Product Condar RE902-C6 06), full-automatic blood gas analyzer (Shanghai Yuyan Scientific Instrument Co., Ltd.; 57984), 96-well plate (Beijing ZEPING Bioscience \& Technologies Co., Ltd.; Nunc 003), high-speed and low-temperature centrifuge (Sichuan Shuke Instrument Co., Ltd.; TGL-16), Enzyme reader (Wuhan ESCN KIT INC. SMR16.1), vortex mixer (Shenzhen Cygen Biotechnology Co., Ltd.; S0200-230V), enzyme-free centrifuge tube (Shanghai Qiming Biological Technology Co., Ltd.; OX02849).

Observation indicators. The postoperative clinical efficacy, general conditions and adverse reactions of the two groups of patients were observed. SBP, DBP and HR were monitored in two groups of patients before anesthesia induction (T0), before laryngeal mask insertion (T1), 30 min after anesthesia (T2), and $3 \mathrm{~h}$ after surgery (T3). The mini-mental state examination (MMSE) scores at $1 \mathrm{~d}$ before operation, 1 and $3 \mathrm{~d}$ after operation were measured to evaluate the cognitive function before and after operation. MMSE scale includes 30 single items in five categories: Orientation, memory, attention and calculation, recall and language. Scores on the MMSE range from 0 to 30, with scores of 27 or higher being traditionally considered normal. Scores less than 27 generally indicate cognitive dysfunction: $21-26$ points are mild impairment, $10-20$ are moderate impairment, and 0-9 are severe impairment (12). 
Table I. Comparison of clinical general data [mean \pm SD, n (\%)].

\begin{tabular}{|c|c|c|c|c|}
\hline Characteristics & Research group $(n=137)$ & Control group $(\mathrm{n}=130)$ & $\chi^{2} / \mathrm{t}$ & P-value \\
\hline Average age (years) & $40.75 \pm 6.88$ & $39.87 \pm 6.31$ & 1.09 & 0.28 \\
\hline Body mass index $\left(\mathrm{kg} / \mathrm{m}^{2}\right)$ & $21.60 \pm 2.50$ & $21.80 \pm 2.30$ & 0.68 & 0.50 \\
\hline History of smoking & & & 0.09 & 0.76 \\
\hline Yes & $37(27.01)$ & $33(25.38)$ & & \\
\hline No & $100(72.99)$ & $97(74.62)$ & & \\
\hline History of drinking & & & 0.03 & 0.86 \\
\hline Yes & $20(14.60)$ & $18(13.85)$ & & \\
\hline No & $117(85.40)$ & $112(86.15)$ & & \\
\hline Education level & & & 0.10 & 0.95 \\
\hline Primary school & $22(16.06)$ & $19(14.62)$ & & \\
\hline Junior high school & $82(59.85)$ & $79(60.77)$ & & \\
\hline Junior college or above & $33(24.09)$ & $32(24.62)$ & & \\
\hline Complications & $117(85.41)$ & $118(90.77)$ & 1.82 & 0.18 \\
\hline Hypertension & $62(45.26)$ & $58(44.62)$ & 0.01 & 0.92 \\
\hline Hyperlipidemia & $55(40.15)$ & $60(46.15)$ & 0.98 & 0.32 \\
\hline Time of operation (min) & $38.81 \pm 8.22$ & $39.12 \pm 7.84$ & 1.32 & 0.75 \\
\hline ASA classification & & & 0.49 & 0.49 \\
\hline Grade I & $119(86.86)$ & $109(83.85)$ & & \\
\hline Grade II & $18(13.14)$ & $21(16.15)$ & & \\
\hline
\end{tabular}

The visual analogue scale (VAS) scores of the two groups of patients were recorded 1 day before operation and 1 day after operation. VAS uses 11 numbers from 0 to 10 to indicate the degree of pain. 0 , no pain, and 10 the worst possible pain. The patient chooses one of the 11 numbers to best describe their current pain. 0 points, no pain; $\leq 3$ points, slight pain that can be tolerated; 4 points to 6 points, pain that affects sleep, but still tolerable; 7 points to 10 points, indicates that the patient has unbearable pain that affects appetite and sleep (13). The blood samples collected at T, T6, T24, T48 and T72 h were used to detect the activity of SOD, CAT and GSH-Px in the serum of patients.

Statistical analysis. SPSS 22.0 (SPSS, Inc.) was used to analyze the data. GraphPad Prism 7 was used for figures (GraphPad Software, Inc.). Count data were expressed as [n (\%)], and Chi-square test was used to compare between groups. Measurement data were presented as mean $\pm \mathrm{SD}$, and t-test was used to compare two groups. ANOVA (parameter) and Tukey were used for multiple comparison. $\mathrm{P}<0.05$ was considered to indicate a statistically significant difference.

\section{Results}

Comparison of clinical general data. The patients between the two groups were compared in terms of age, body mass index, history of smoking and drinking, education level and complications $(\mathrm{P}>0.05)$, which was comparable (Table I).

Comparison of clinical efficacy. Statistical analysis showed that the total effective rate of the research group (91.24\%) was not significantly different from that of the control group $(90.77 \%)(\mathrm{P}>0.05$; Table II).

Comparison of general conditions before and after operation. The general conditions of patients in the two groups before and after surgery were recorded (Table III). The body movement, dysphoria during the awakening period, eye-opening time and extubation time in the research group were lower than those in the control group $(\mathrm{P}<0.05)$, there was no significant difference in PONV between the two groups $(\mathrm{P}>0.05)$, while hypotension during surgery in the research group was significantly higher than that in the control group $(\mathrm{P}<0.05)$.

Comparison of hemodynamic indicators. Before anesthesia, there was no significant difference in the hemodynamic indicators of the two groups $(\mathrm{P}>0.05)$. The levels of SBP and $\mathrm{DBP}$ were significantly lower at $\mathrm{T} 1$ and $\mathrm{T} 2$ than those at $\mathrm{T} 0$ $(\mathrm{P}<0.05)$, and those at $\mathrm{T} 1$ were lower than those at $\mathrm{T} 2(\mathrm{P}<0.05)$. There was no significant difference in the levels of SBP and DBP between T3 and T0 ( $>>0.05)$, while the levels of SBP and DBP at T1 and T2 in the control group were significantly higher than those in the research group $(\mathrm{P}<0.05)$. There was no difference in HR in the control group at T1, T2, T3 and T0 $(\mathrm{P}>0.05), \mathrm{HR}$ in the research group at $\mathrm{T} 1$ and $\mathrm{T} 2$ was lower than that at T0 and corresponding time points of the control group $(\mathrm{P}<0.05)$, and $\mathrm{HR}$ at $\mathrm{T} 3$ and $\mathrm{T} 0$ had no significant difference $(\mathrm{P}>0.05$; Table IV).

Comparison of adverse reactions after surgery. Comparison of postoperative adverse reactions of patients (Table V) showed that adverse reactions of patients in both groups were relieved 
Table II. Comparison of clinical efficacy [n (\%)].

\begin{tabular}{lccccc}
\hline Group & Cure & Markedly effective & Effective & Ineffective & Total effective rate \\
\hline Research group $(\mathrm{n}=137)$ & $38(27.74)$ & $52(37.96)$ & $35(25.55)$ & $12(8.75)$ & $125(91.24)$ \\
Control group $(\mathrm{n}=130)$ & $36(27.70)$ & $47(36.15)$ & $34(26.15)$ & $13(10.00)$ & $117(90.00)$ \\
$\chi^{2}$ & - & - & - & - & 0.06 \\
P-value & - & - & - & - & 0.81 \\
\hline
\end{tabular}

Table III. Comparison of general conditions before and after surgery [mean $\pm \mathrm{SD}, \mathrm{n}(\%)]$.

\begin{tabular}{lccccc}
\hline Group & $\begin{array}{c}\text { Body movement } \\
\text { during surgery }\end{array}$ & $\begin{array}{c}\text { Hypotension during } \\
\text { surgery }\end{array}$ & $\begin{array}{c}\text { Extubation } \\
\text { time (min) }\end{array}$ & $\begin{array}{c}\text { Dysphoria during } \\
\text { the awakening period }\end{array}$ & $\begin{array}{c}\text { Eye-opening } \\
\text { time (min) }\end{array}$ \\
PONV
\end{tabular}

Intraoperative hypotension: blood pressure decrease by $>20 \%$ before anesthesia or systolic blood pressure is $<80 \mathrm{mmHg}$. PONV, postoperative nausea and vomiting.

after symptomatic treatment, and there was no difference between the research group and the control group $(\mathrm{P}>0.05)$.

Comparison of MMSE score. According to the MMSE scores of patients in the two groups (Fig. 1), there was no significant difference between the research group $(26.63 \pm 1.74)$ and the control group $(25.65 \pm 1.23)$ on the first day before surgery $(\mathrm{P}>0.05)$. On the first day after surgery, both groups decreased, but the score in the research group $(20.92 \pm 0.88)$ was higher than that in the control group $(17.83 \pm 0.98)(\mathrm{P}<0.05)$. Three days after surgery, the research group $(26.95 \pm 1.63)$ and the control group $(26.70 \pm 1.48)$ returned to normal $(\mathrm{P}>0.05)$, there was no significant difference between the groups $(\mathrm{P}>0.05)$.

Comparison of VAS score. According to the VAS scores of patients in the two groups (Fig. 2), there was no significant difference between the research group $(4.89 \pm 0.66)$ and the control group $(4.98 \pm 0.70)$ before surgery $(\mathrm{P}>0.05)$; after surgery, both groups were significantly lower than those before surgery $(\mathrm{P}<0.05)$, while the research group $(2.17 \pm 0.59)$ was significantly lower than that of the control group $(3.17 \pm 0.59)$ $(\mathrm{P}<0.05)$.

Comparison of serum SOD, CAT and GSH-Px activities. The activities of SOD, CAT and GSH-Px of patients in the two groups at $\mathrm{T} 24$ and $\mathrm{T} 48 \mathrm{~h}$ were lower than those at $\mathrm{T}(\mathrm{P}<0.05)$, while the activities of SOD and GSH-Px in the control group at $\mathrm{T} 72 \mathrm{~h}$ were still lower than those at $\mathrm{T}(\mathrm{P}<0.05)$, and the CAT activity returned to normal $(\mathrm{P}>0.05)$. In the research group, when SOD at T72 h was lower than those at $\mathrm{T}(\mathrm{P}<0.05)$, CAT and GSH-Px activities returned to normal $(\mathrm{P}>0.05)$. The activities of SOD, CAT and GSH-Px in the control group at T6 $\mathrm{h}$ were lower than those at $\mathrm{T}(\mathrm{P}<0.05)$. When the GSH-Px activity at $\mathrm{T} 6 \mathrm{~h}$ was lower than that at $\mathrm{T}(\mathrm{P}<0.05), \mathrm{SOD}$ and
CAT had no significant difference at $\mathrm{T}(\mathrm{P}>0.05)$. The SOD activity in the control group was significantly lower than that in the research group from T6 to $\mathrm{T} 72 \mathrm{~h}(\mathrm{P}<0.05)$, while the CAT activity in the control group was significantly lower than that in the research group at $\mathrm{T} 24 \mathrm{~h}(\mathrm{P}<0.05)$. Compared with the research group, the GSH-Px activity in the control group decreased significantly from T6 to T48 h ( $\mathrm{P}<0.05$; Fig. 3).

\section{Discussion}

In surgical operations, ideal anesthesia requires stable hemodynamics and rapid anesthesia induction. Patients should wake up quickly and completely after withdrawal of drugs, without dysphoria after surgery, respiratory depression and drug residue (14). At present, the clinical application of intravenous anesthesia is gradually paid attention to. In clinical general anesthesia, propofol combined with remifentanil is mainly used for drug compatibility, which has the advantages of rapid anesthesia and postoperative recovery $(15,16)$. Propofol $(17)$ can be rapidly removed from the central ventricle through liver metabolism and renal excretion, while remifentanil $(18,19)$ can be rapidly degraded by non-specific esterase, which was advantageous in rapid action, short action time, rapid removal without accumulation during continuous infusion. Inhalation anesthesia also has advantages including quick effect and discharge, little influence on circulation and respiration, and non-invasive administration. Therefore, intravenous compound anesthesia is often used in order to give full play to the characteristics of various drugs and achieve stable anesthesia, less physiological disturbance and side effects as well as quick recovery (20).

In this study, it was found that the total effective rate of the research group $(91.24 \%)$ was not significantly different from that of the control group $(90.77 \%)(\mathrm{P}>0.05)$. In the 
Table IV. Comparison of hemodynamic indicators at different time points (mean \pm SD).

\begin{tabular}{llccccrr}
\hline Indicators & \multicolumn{1}{c}{ Group } & T0 & T1 & T2 & T3 & F & P-value \\
\hline SBP $(\mathrm{mmHg})$ & Research group & $121.4 \pm 10.7$ & $106.5 \pm 9.6^{\mathrm{a}}$ & $110.5 \pm 9.4^{\mathrm{a}}$ & $119.5 \pm 9.1$ & 74.88 & $<0.01$ \\
& Control group & $122.2 \pm 10.9$ & $115.3 \pm 9.2^{\mathrm{a}}$ & $119.9 \pm 9.3$ & $120.4 \pm 9.3$ & 11.92 & $<0.01$ \\
& $\mathrm{t}$ & 0.61 & 7.64 & 8.21 & 0.80 & & \\
& P-value & 0.55 & $<0.01$ & $<0.01$ & 0.42 & & \\
DBP (mmHg) & Research group & $81.5 \pm 8.5$ & $73.5 \pm 7.4^{\mathrm{a}}$ & $75.9 \pm 7.3^{\mathrm{a}}$ & $80.2 \pm 7.9$ & 31.27 & $<0.01$ \\
& Control group & $82.6 \pm 7.6$ & $78.7 \pm 6.9^{\mathrm{a}}$ & $81.1 \pm 6.4$ & $81.3 \pm 7.2$ & 6.93 & $<0.01$ \\
& $\mathrm{t}$ & 0.11 & 5.93 & 6.18 & 1.19 & & \\
& P-value & 0.27 & $<0.01$ & $<0.01$ & 0.24 & & $<0.01$ \\
HR (times/min) & Research group & $75.4 \pm 7.0$ & $69.8 \pm 6.5^{\mathrm{a}}$ & $71.8 \pm 6.6^{\mathrm{a}}$ & $75.4 \pm 6.9$ & 23.12 & 0.31 \\
& Control group & $76.0 \pm 7.2$ & $74.5 \pm 7.0$ & $75.2 \pm 6.9$ & $75.8 \pm 7.1$ & 1.19 & \\
& $\mathrm{t}$ & 0.69 & 5.69 & 4.12 & 0.47 & & \\
& P-value & 0.49 & $<0.01$ & $<0.01$ & 0.64 & & \\
\hline
\end{tabular}

${ }^{\mathrm{a}} \mathrm{P}<0.05$, compared with with T0 in the same group. SBP, systolic blood pressure; DBP, diastolic blood pressure; HR, heart rate.

Table V. Comparison of postoperative adverse reactions [n (\%)].

\begin{tabular}{|c|c|c|c|c|c|c|c|}
\hline Group & Dizziness & Cough & Insomnia & Headache & Rash & Inappetite & $\begin{array}{c}\text { Incidence rate } \\
\text { of adverse reactions }\end{array}$ \\
\hline Research group $(n=137)$ & $4(2.92)$ & $2(1.46)$ & $6(4.38)$ & $5(3.65)$ & $0(0.00)$ & $4(2.92)$ & $21(15.33)$ \\
\hline Control group $(n=130)$ & $6(4.62)$ & $6(4.62)$ & $6(4.62)$ & $3(2.31)$ & $1(0.75)$ & $4(3.08)$ & $26(20.00)$ \\
\hline$\chi^{2}$ & 0.53 & 2.29 & 0.01 & 0.41 & 1.06 & 0.01 & 1.00 \\
\hline P-value & 0.47 & 0.13 & 0.93 & 0.52 & 0.30 & 0.94 & 0.32 \\
\hline
\end{tabular}

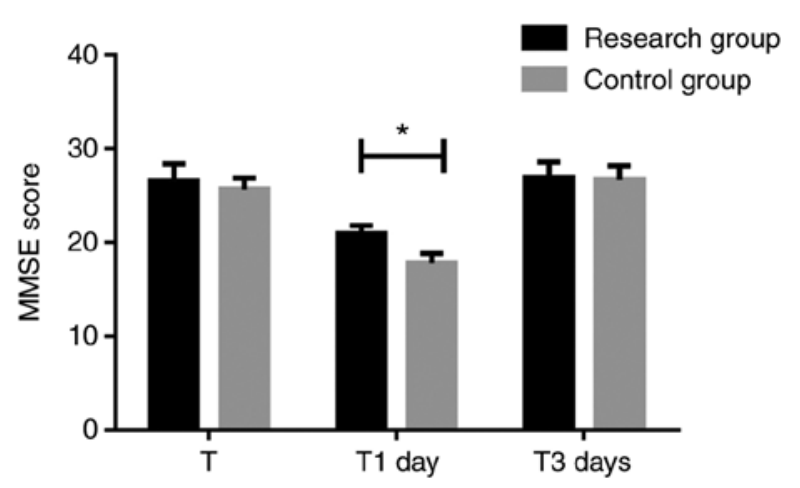

Figure 1. MMSE scores of patients in the two groups before and after surgery. In the MMSE scores of patients, there was no significant difference compared with those of the two groups one day before surgery; both groups decreased one day after surgery, but the research group was higher than the control group; three days after surgery, both groups recovered to normal, and there was no significant difference between the two groups. ${ }^{*} \mathrm{P}<0.05$, for the comparison between the two groups. MMSE, mini-mental state examination.

randomized controlled trial of propofol intravenous anesthesia and isoflurane inhalation anesthesia by Visser et al (21), the total effective rate of the two anesthesia methods was not significantly different, which was similar to our results. Therefore, we speculated that anesthesia methods had certain influence on clinical efficacy, but the main influence was still

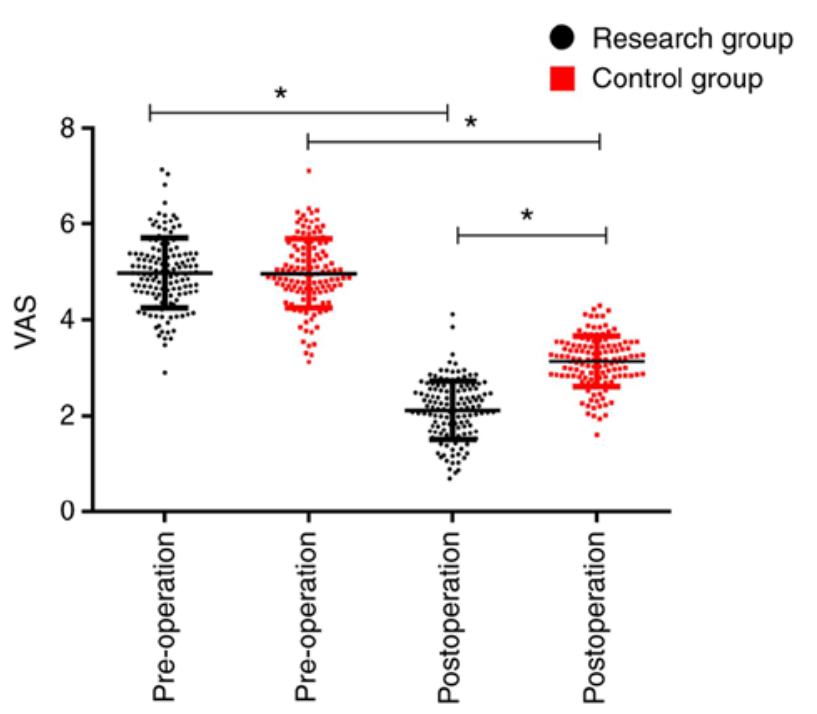

Figure 2. VAS scores of patients in the two groups before and after surgery. In VAS scores of patients, there was no significant difference between the two groups before and after surgery, both groups after surgery were significantly lower than those before surgery, and the research group was significantly lower than the control group. ${ }^{*} \mathrm{P}<0.05$, for the comparison between the two groups. VAS, visual analogue scale.

on the condition of patients, operation methods and other reasons. In this study, the number of hypotension during 

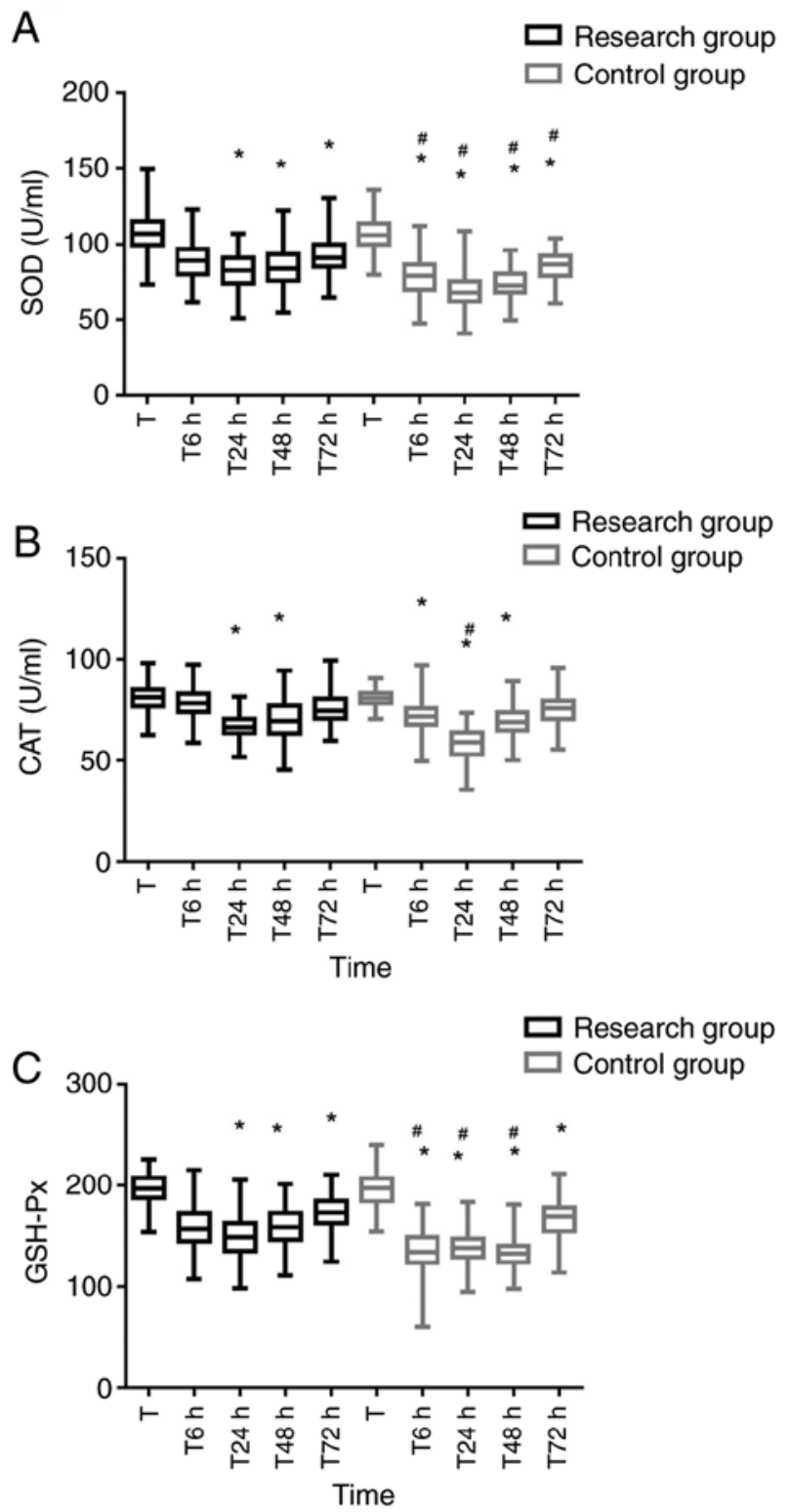

Figure 3. Comparison of serum SOD, CAT and GSH-Px activities at different time points. (A) Comparison of serum SOD activities at different time points. (B) comparison of serum CAT activities at different time points. (C) Comparison of serum GSH-Px activities at different time points. SOD, CAT and GSH-Px activities of patients in the two groups were lower at T24 and T48 $\mathrm{h}$ than those at T, while SOD and GSH-Px activities in the control group were still lower at $\mathrm{T} 72 \mathrm{~h}$ than those at T. The SOD in the research group was lower at T72 h than that at T, and the activities of SOD, CAT and GSH-Px in the control group at T6 $\mathrm{h}$ were lower than those at T. When GSH-Px activity in the research group was lower at T6 $\mathrm{h}$ than that at T, SOD activity in the control group was significantly lower than that in the research group from T6 h to T72 h, while CAT activity in the control group was significantly lower than that in the research group at T24 $\mathrm{h}(\mathrm{P}<0.05)$. The GSH-Px activity of the control group decreased significantly from $\mathrm{T} 6$ to $\mathrm{T} 48 \mathrm{~h}$ compared with those in the research group $(\mathrm{P}<0.05)$. ${ }^{\#} \mathrm{P}<0.05$, compared with the research group at the same time point; ${ }^{*} \mathrm{P}<0.05$, compared with T0. SOD, superoxide dismutase; CAT, catalase; GSH-Px, glutathione peroxidase.

surgery in the research group was also significantly higher than that in the control group $(\mathrm{P}<0.05)$. In the research group before and after surgery, body movement during surgery, dysphoria during the awakening period, eye-opening time and extubation time were lower than those of the control group $(\mathrm{P}<0.05)$. Kim et al (22) compared remifentanil+propofol and remifentanil+sevoflurane, and the results revealed that the propofol group had a shorter awakening time and extubation time. In the research of Scott et al (23), it was found that although propofol and remifentanil had different pharmacodynamic effects, the two drugs interacted in realizing loss of consciousness and analgesia. The use of analgesia in total intravenous anesthesia can produce an effect of saving propofol and possibly minimize the inhibition of EEG activity. Presumably, the research group used propofol combined with remifentanil, which had short duration of stay in vivo and short recovery time after surgery, so the research group had shorter recovery time than the control group. In a previous study, the increase of incidence rate of PONV was related to inhalation anesthetics (24), however, there was no significant difference in the incidence rate of PONV between the research group and the experimental group in this study $(\mathrm{P}>0.05)$, and there were certain differences between this study and their research, which is why further research was needed.

The SBP, DBP and HR of patients all returned to normal levels $3 \mathrm{~h}$ after anesthesia. Mohaghegh et al (25) compared the effects of propofol intravenous anesthesia and isoflurane inhalation anesthesia on postoperative pain of inguinal hernia, and found that there was no significant difference in SBP, DBP and HR between the two groups after induction, during intubation, after intubation and extubation $(\mathrm{P}>0.05)$. In our study, although there was no significant difference in SBP, DBP, HR at T3 and T0 ( $>>0.05)$, there was a significant difference in SBP, DBP in the two groups between T1, T2 and T0 $(\mathrm{P}<0.05)$. HR in the research group at $\mathrm{T} 1$ and $\mathrm{T} 2$ was lower than those at T0 $(\mathrm{P}<0.05)$, it was presumed that propofol combined with remifentanil was used in the research group, while Mohaghegh et al (25) just used propofol, because remifentanil might make hemodynamic fluctuation more obvious, so further detailed study was needed. Compared with the control group, the hemodynamic fluctuation of patients in the research group was more obvious. The levels of SBP and DBP in the two groups were significantly lower at T1 and T2 than those at $\mathrm{T} 0(\mathrm{P}<0.05)$, and those at $\mathrm{T} 1$ were lower than those at T2 $(\mathrm{P}<0.05)$, while the levels of SBP and DBP at T1 and $\mathrm{T} 2$ in the control group were significantly higher than those in the research group $(\mathrm{P}<0.05)$. HR of research group at $\mathrm{T} 1$ and $\mathrm{T} 2$ was lower than that at T0 and corresponding time in control group $(\mathrm{P}<0.05)$. The number of hypotension during surgery was significantly higher than that of the control group, because both drugs could inhibit the circulation of the body to a certain extent, the SBP, DBP and HR in the research group were generally lower than those in the control group, which was prone to hypotension during surgery and bradycardia (26).

In this study, there was no difference in adverse reactions between the research group and the control group ( $\mathrm{P}>0.05)$; after symptomatic treatment, the adverse reactions of patients in both groups were relieved. MMSE scores of patients in the two groups decreased one day after surgery, but the score was higher in the research group than that in the control group $(\mathrm{P}<0.05)$. The scores of both groups returned to normal level three days after surgery $(\mathrm{P}>0.05)$. Yu (27) confirmed that the $12 \mathrm{~h}$ MMSE scores of patients 1,6 and $12 \mathrm{~h}$ after surgery were significantly lower than those before surgery $(\mathrm{P}<0.05)$ by analyzing the effect of propofol and sevoflurane combined anesthesia on cognitive function of elderly patients 
undergoing total thoracic surgery. The score of propofol group was higher than that in the sevoflurane group $(\mathrm{P}<0.05)$, which was consistent with the results of the present study. However, patients returned to normal after three days of follow-up, and it was presumed that propofol combined with remifentanil or sevoflurane would both affect the cognitive function of patients to a certain extent during surgery, while propofol combined with remifentanil had relatively little effect on the cognitive function, but neither anesthesia have permanent effect on it. In this study, the VAS scores of the two groups were significantly lower after surgery than those before surgery $(\mathrm{P}<0.05)$, while the score of research group was significantly lower than that in the control group $(\mathrm{P}<0.05)$. Research of Elbakry et al (28) on the influence of inhalation (desflurane) and total intravenous anesthesia (propofol and dexmedetomidine) on postoperative rehabilitation of morbid obesity patients after laparoscopic sleeve gastrectomy showed the intravenous anesthesia group had a lower postoperative VAS $(\mathrm{P}<0.001)$, which was consistent with the results of this study. It indicated that when propofol or sevoflurane was used during surgery, it could alleviate the need for postoperative analgesia to a certain extent, as well as the need for analgesia of patients undergoing propofol anesthesia.

In the present study, the activities of SOD, CAT and GSH-Px of patients in the two groups were lower at T24 and $\mathrm{T} 48 \mathrm{~h}$ than those at $\mathrm{T}(\mathrm{P}<0.05)$, while the activities of SOD and GSH-Px in the control group at T72 h were still lower than those at $\mathrm{T}(\mathrm{P}<0.05)$. In the research group, SOD was lower at $\mathrm{T} 72 \mathrm{~h}$ than that at $\mathrm{T}(\mathrm{P}<0.05)$. The activities of SOD, CAT and GSH-Px in the control group at T6 $\mathrm{h}$ were lower than those at $\mathrm{T}(\mathrm{P}<0.05)$. GSH-Px activity in the research group at T6 $\mathrm{h}$ was lower than that at $\mathrm{T}(\mathrm{P}<0.05)$. SOD activity in the control group was significantly lower than that in the research group from T6 to $\mathrm{T} 72 \mathrm{~h}(\mathrm{P}<0.05)$, while CAT activity in the control group was significantly lower than that in the research group at T24 h $(\mathrm{P}<0.05)$. The GSH-Px activity of the control group decreased significantly from T6 to T48 h compared with that in the research group $(\mathrm{P}<0.05)$. In a previous study (29) propofol inhibited mitochondrial dysfunction and OS of liver I/R, propofol instead of sevoflurane prevented mitochondrial dysfunction and OS by limiting the activation of HIF-1 $\alpha$ in liver ischemia/reperfusion injury. The SOD activity in the research group was significantly higher than that in the control group, which was in accordance with the results of this study, indicating that propofol had stronger inhibition on OS response of patients compared with sevoflurane. Since there was no significant difference between the research group and the control group at $\mathrm{T} 72 \mathrm{~h}$ and patients in the two groups gradually recovering over time, it was presumed that sevoflurane also had certain antagonism to OS.

This study comprehensively explored the clinical efficacy of breast segmental surgery under intravenous anesthesia and inhalation anesthesia and the changes of patients' hemodynamics and OS response, with the aim to provide certain reference for clinical research. However, the specific mechanism of intravenous anesthesia and the influence in different surgeries need to be further explored, the relationship between clinical pathological factors and anesthesia methods needs to be analyzed by multiple factors, and the application of intravenous anesthesia in clinical practice needs to be further studied, to explore the influence of various factors on clinical efficacy, to provide reference for more accurate judgment of patients.

In summary, intravenous anesthesia can reduce the occurrence of postoperative adverse reactions, improve sedation and analgesia, and make patients wake up quickly and stably after surgery, and recover cognitive function and OS rapidly, but it has a great impact on hemodynamics during surgery. Attention should be paid to maintaining hemodynamic stability and avoiding occurrence of hypotension and bradycardia during surgery. Furthermore, it is a better anesthesia method for breast segmental surgery.

\section{Acknowledgements}

Not applicable.

\section{Funding}

No funding was received.

\section{Availability of data and materials}

The datasets used and/or analyzed during the present study are available from the corresponding author on reasonable request.

\section{Authors' contributions}

$\mathrm{XY}, \mathrm{YZ}$ and $\mathrm{HH}$ conceived and designed the study, and drafted the manuscript. XY, XZ, SS and HH collected, analyzed and interpreted the experimental data. $\mathrm{YZ}$ revised the manuscript for important intellectual content. All authors read and approved the final manuscript.

\section{Ethics approval and consent to participate}

The study was approved by the Ethics Committee of Chun'an First People's Hospital (Hangzhou, China). Signed written informed consents were obtained from the patients and/or guardians.

\section{Patient consent for publication}

Not applicable.

\section{Competing interests}

The authors declare that they have no competing interests.

\section{References}

1. Mahmoud M and Mason KP: Recent advances in intravenous anesthesia and anesthetics. F1000Res 7: F1000 Faculty Rev-470 F1000 Faculty Rev-470, 2018.

2. Absalom AR and Mason KP: Total intravenous anesthesia and target controlled infusions. 2017 doi: 10.1007/978-3-319-47609-4.

3. Poprac P, Jomova K, Simunkova M, Kollar V, Rhodes CJ and Valko M: Targeting free radicals in oxidative stress-related human diseases. Trends Pharmacol Sci 38: 592-607, 2017.

4. Athar M: Oxidative stress and experimental carcinogenesis. Indian J Exp Biol 40: 656-667, 2002.

5. Ishii T, Yasuda K, Akatsuka A, Hino O, Hartman PS and Ishii N: A mutation in the SDHC gene of complex II increases oxidative stress, resulting in apoptosis and tumorigenesis. Cancer Res 65: 203-209, 2005. 
6. Ray G, Batra S, Shukla NK, Deo S, Raina V, Ashok S and Husain SA: Lipid peroxidation, free radical production and antioxidant status in breastcancer. Breast Cancer Res Treat 59: $163-170,2000$

7. Saygili EI, Akcay T, Konukoglu D and Papilla C: Glutathione and glutathione-related enzymes in colorectal cancer patients. J Toxicol Environ Health A 66: 411-415, 2003.

8. Höhn A, Weber D, Jung T, Ott C, Hugo M, Kochlik B, Kehm R, König J, Grune T and Castro JP: Happily (n)ever after: Aging in the context of oxidative stress, proteostasis loss and cellular senescence. Redox Biol 11: 482-501, 2017.

9. Tain RW, Scotti AM, Li W, Zhou XJ and Cai K: Imaging short-lived reactive oxygen species (ROS) with endogenous contrast MRI. J Magn Reson Imaging 47: 222-229, 2018.

10. Davis SM and Pennypacker KR: Targeting antioxidant enzyme expression as a therapeutic strategy for ischemic stroke. Neurochem Int 107: 23-32, 2017.

11. Ahmed RG: The physiological and biochemical effects of diabetes on the balance between oxidative stress and antioxidant defense system. Med J Islamic World Acad Sci 15: 31-42, 2005.

12. Wong A, Black SE, Yiu SYP, Au LWC, Lau AYL, Soo YOY, Chan AYY, Leung TWH, Wong LKS, Kwok TCY, et al: Converting MMSE to MoCA and MoCA 5-minute protocol in an educationally heterogeneous sample with stroke or transient ischemic attack. Int J Geriatr Psychiatry 33: 729-734, 2018.

13. Le May S, Ballard A, Khadra C, Gouin S, Plint AC, Villeneuve E, Mâsse B, Tsze DS, Neto G, Drendel AL, et al: A comparison of the psychometric properties of three pain scales used in the pediatric emergency department: VAS, FPS-R and CAS Pain 159: 1508-1517, 2018.

14. Soleimani A, Heidari N, Habibi MR, Kiabi FH, Khademloo M, Emami Zeydi A and Sohrabi FB: Comparing hemodynamic responses to diazepam, propofol and etomidate during anesthesia induction in patients with left ventricular dysfunction undergoing coronary artery bypass graft surgery: A double-blind, randomized clinical trial. Med Arch 71: 198-203, 2017.

15. Masjedi M,Zand F, Kazemi AP and Hoseinipour A: Prophylactic effect of ephedrine to reduce hemodynamic changes associated with anesthesia induction with propofol and remifentanil. J Anaesthesiol Clin Pharmacol 30: 217-221, 2014.

16. Cho YJ, Kim TK, Hong DM, Seo JH, Bahk JH and Jeon Y: Effect of desflurane-remifentanil vs. Propofol-remifentanil anesthesia on arterial oxy genation during one-lung ventilation for thoracoscopic surgery: A prospective ran domized trial. BMC Anesthesiol 17: 9, 2017.

17. Feng AY, Kaye AD, Kaye RJ, Belani K and Urman RD: Novel propofol derivatives and implications for anesthesia practice. J Anaesthesiol Clin Pharmacol 33: 9-15, 2017.

18. Battershill AJ and Keating GM: Remifentanil: A review of its analgesic and sedative use in the intensive care unit. Drugs 66 365-385, 2006

19. Kisilcwicz M, Rosenherg H and Vaillancourt C: Remifentanil for procedural sedation; a systematic review of the literature. Emerg Mcd J 34: 294-301, 2017.
20. Xu R, Zhou S, Yang J, Li H, Zhang Q, Zhang G, Xu S and Peng Q: Total intravenous anesthesia produces outcomes superior to those with combined intravenous-inhalation anesthesia for laparoscopic gynecological surgery at high altitude. J Int Med Res 45: 246, 2017.

21. Visser K, Hassink EA, Bonsel GJ, Moen J and Kalkman C: Randomized controlled trial intravenous anesthesia with propofol versus inhalation anesthesia with isoflurane-nitrous oxide. Anesthesiology 95: 616-626, 2001.

22. KimH,ChoiSH,Choi YS,Lee JH,KimNO and Lee JR: Comparison of the antitussive effect of remifentanil during recovery from propofol and sevoflurane anaesthesia. Anaesthesia 67: 765-770, 2012.

23. Scott HB, Choi SW, Wong GTC and Irwin MG: The effect of remifentanil on propofol requirements to achieve loss of response to command vs. loss of response to pain. Anaesthesia 72: 479-487, 2017.

24. Park SK and Cho EJ: A randomized controlled trial of two different interventions for the prevention of postoperative nausea and vomiting; total intravenous anaesthesia using propofol and remifentanil versus prophylactic palonosetron with inhalational anaesthesia using sevofluraef-nitrous oxide. J Int Med Res 39: 1808-1815, 2011.

25. Mohaghegh T, Yazdi B, Norouzi A, Fateh S, Modir H and Mohammadbeigi A: Effect of intravenous anesthesia with propofol versus isoflurane inhalation anesthesia in postoperative pain of inguinal herniotomy: A randomized clinical trial. Med Gas Res 7: 86-92, 2017

26. Oncul S, Gaygusuz EA, Yilmaz M, Terzi H and Balci C: Comparison of ketamine-propofol and remifentanil in terms of hemodynamic variables and patient satisfaction during monitored anaesthesia care. Anasthesiol Intensive Ther 48: 116-121, 2016.

27. $\mathrm{Yu} \mathrm{W:} \mathrm{Anesthesia} \mathrm{with} \mathrm{propofol} \mathrm{and} \mathrm{sevoflurane} \mathrm{on} \mathrm{postop-}$ erative cognitive function of elderly patients undergoing general thoracic surgery. Pak J Pharm Sci 30: 1107-1110, 2017.

28. Elbakry AE, Sultan WE and Ibrahim E: A comparison between inhalational (Desflurane) and total intravenous anaesthesia (Propofol and dexmedetomidine) in improving postoperative recovery for morbidly obese patients undergoing laparoscopic sleeve gastrectomy: A double-blinded randomised controlled trial. J Clin Anesth 45: 6-11, 2018.

29. Bellanti F, Mirabella L, Mitarotonda D, Blonda M, Tamborra R, Cinnella G, Fersini A, Ambrosi A, Dambrosio M, Vendemiale G and Serviddio G: Propofol but not sevoflurane prevents mitochondrial dysfunction and oxidative stress by limiting HIF-1a activation in hepatic ischemia/reperfusion injury. Free Radic Biol Med 3: 323-333, 2016.

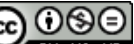

This work is licensed under a Creative Commons Attribution-NonCommercial-NoDerivatives 4.0 International (CC BY-NC-ND 4.0) License. 